\title{
印章结构对微接触印刷制备导电网格的影响
}

\author{
辛智青，李 修，姬 磊，李泽涛，项飞翔，刘世丽，李路海 \\ (北京印刷学院 印刷与包装工程学院，北京市印刷电子工程技术研究中心，北京 102600)
}

摘 要: 为了在 PET 基底上制备精细的透明网格状导电图案, 本工作利用微接触印刷高精度的优势, 分别采用线条 结构和网格结构的印章转印银纳米粒子导电油墨, 分析了其转印过程, 并讨论了印章结构对网格图案性能的影响。 结果表明: 采用线条结构的印章可真实还原印章设计尺寸, 避免了线条的扩展, 有利于提高网格图案的透光率; 同 时, 在交叉处墨层较厚可提高导电性。而采用网格结构印章可一次转移快速获得网格图案, 但由于印章网格上相邻 线条之间液桥的形成会使图案线宽增加, 降低其透光率; 采用间距较大的印章可使液桥断裂, 得到边缘光滑的网 格图案, 但间距增大导致单位面积导电路径减少, 降低其导电性。总之, 采用线条结构印章有利于获得性能较好的 网格图案, 但两次转移过程相对复杂, 需用时间较长。

关 键 词: 微接触印刷; 网格图案; 导电油墨; 银纳米粒子

中图分类号: 0647 文献标识码: A

\section{Stamp Structure on the Conductive Grid Patterns Prepared by Microcontact Printing}

\author{
XIN Zhi-Qing, LI Xiu, JI Lei, LI Ze-Tao, XIANG Fei-Xiang, LIU Shi-Li, LI Lu-Hai
}

(School of Printing \& Packaging, Beijing Institute of Graphic Communication, Beijing Printed Electronics Engineering Technology Research Center, Beijing 102600, China)

\begin{abstract}
Conductive grid patterns with fine line were achieved on the PET substrate via microcontact printing silver nanoparticle ink for its high resolution advantage. Conductive grids were patterned and compared by using the stamps with either line- or grid-structured patterns. When using line-structured stamp, the line width of the grid pattern was nearly the same as that on the stamp and the printed line edge was also smooth. The extra thickness at the cross of the grid which was due to two sequential stamp operations, was beneficial for higher conductivity. When using the grid-structured stamp, the conductive grid was acquired by only one stamp operation. However, the line width was broader than designed on the stamp and the line edge was poorer amid to liquid bridges formed between two adjacent lines on the stamp. The situation improved for the liquid bridge was easy to rupture when the line spacing of the grid line became bigger, but the conductivity was lowered as fewer conductive paths within the unit area. Hence, it is beneficial to acquire grid patterns using line-structured stamp, but the process is complicated and it will take longer time to complete two successive transfer processes.
\end{abstract}

Key words: microcontact printing; grid patterns; conductive ink; silver nanoparticles

收稿日期：2016-10-19; 收到修改稿日期：2016-12-03

基金项目: 国家自然科学基金(21403014,61474144); 北京印刷学院校级一般科研项目(Eb201501); 绿色印刷与出版技术 协同创新中心(2011); 北京高等学校高水平人才交叉培养实培计划; 北京印刷学院重点项目(Ea201501)

National Natural Science Foundation of China $(21403014,61474144)$; General Project of Beijing Institute of Graphic Communication (Eb201501); Collaborative Innovation Center of Green Printing \& Publishing Technology (2011); Gross Training Plan of High Level Talents in Beijing Colleges and Universities; Key Project of Beijing Institute of Graphic Commication (Ea201501)

作者简介: 辛智青(1980-), 男, 博士，讲师. E-mail: zhiqingxin@bigc.edu.cn

通讯作者: 李路海, 教授. E-mail: liluhai@bigc.edu.cn 
相对于传统硅基电子, 印刷电子产品以其大 面积、柔性、轻薄等特点而受到广泛关注。透明 电极作为印刷电子产品之一, 具有良好的机械 性、透光性和导电性, 可用于显示 ${ }^{[1]}$ 、触摸屏 ${ }^{[2]}$ 和太阳能电池 ${ }^{[3]}$ 等领域。纳米材料可溶液化处理, 促使采用印刷纳米线 ${ }^{[4]}$ 、纳米粒子 ${ }^{[5]}$ 、导电聚合 物 ${ }^{[6]}$ 及这些材料的混合物 ${ }^{[7]}$ 制备透明电极成为可 能, 例如, 通过柔印 ${ }^{[8]}$ 、凹印 ${ }^{[9]} \mathrm{c}^{2}$ 纳米粒子导电油 墨的方法实现低成本制备金属网格电极。然而, 传统印刷技术仅仅具有 $50 \sim 100 \mu \mathrm{m}$ 的分辨率, 无 法满足 OLED 显示要求。分辨率成为制约网格状 电极应用的瓶颈之一, 开发新的印刷技术已成为 必然, 如纳米压印已经被用于制备网格电极 ${ }^{[10]}$, 但其成本高。

微接触印刷(microcontact printing, $\mu \mathrm{CP}$ ) 作为软刻 蚀(soft lithography)技术的一个分支, 1994 年由哈佛大 学 Whitesides 教授 ${ }^{[11]}$ 发明, 他们采用弹性印章 PDMS(聚二甲基硅氧烷)蘸取硫醇分子进行转印, 结 合自组装技术和刻蚀技术, 得到几百纳米的图案, 相 对于传统印刷技术具有高分辨率的优势。随着纳米技

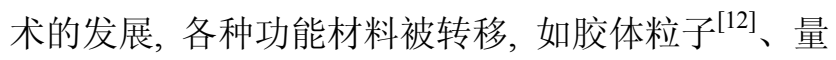
子点 ${ }^{[13]}$ 、金属催化剂 ${ }^{[14]}$ 、金属纳米粒子 ${ }^{[15]}$ 和聚合物 ${ }^{[16]}$ 等, 并用微接触印刷方式制备有机薄膜晶体管 ${ }^{[17]}$ 。 当转移小分子时, 由于其具有自组装特性仅通过 扩散就可从 PDMS 印章快速转移到基底上; 而微 纳米颗粒自组装特性相对弱, 转移微纳米颗粒与转 移小分子有很大差异, 控制印章表面能、墨层与印章 的粘附力、转印条件对于油墨的转移非常重要 ${ }^{[18]}$ 。 在前期实验中, 本课题组研究了微接触印刷转印 银纳米粒子导电油墨的条件 ${ }^{[19]}$ 。本工作借助微接触 印刷高精度的优势, 分别采用线条结构和网格结 构的印章转印银纳米粒子导电油墨, 在 PET 基底 上获得导电网格图案, 并讨论其转印过程, 对比分 析网格图案的性能。

\section{1 实验方法}

\section{1 印章的制备}

印章材料通常选用聚二甲基硅氧烷, 将固化 剂和预聚物 (Sylgard 184, Dow corning) 按质量比 1：10 混合搅拌, 随后离心去除气泡 $(3000 \mathrm{r} / \mathrm{min}$, $10 \mathrm{~min}$ ), 并浇筑在预先氟硅烷处理的硅模板上加 热固化 $\left(80^{\circ} \mathrm{C}, 3 \mathrm{~h}\right)$, 最后从模板上将 PDMS 膜揭下 来, 裁成 $1 \mathrm{~cm} \times 1 \mathrm{~cm}$ 备用。线条结构和网格结构的 印章分别用于转印(线条间距分别为 $10 、 20 、 40 \mu \mathrm{m}$,
高度固定为 $20 \mu \mathrm{m}$, 宽度固定为 $10 \mu \mathrm{m}$ )制备网格图 案, 印章如图 1(a)所示。

\section{2 微接触印刷}

印刷之前分别用丙酮、乙醇、去离子水将玻璃 片、PDMS 印章、PET 基底超声清洗, 然后 $\mathrm{N}_{2}$ 吹干。 采用自制的转印设备印刷实验室自制的银纳米粒子 导电油墨 ${ }^{[20]}$ (含 $20 \mathrm{wt} \%$ 银纳米粒子、 $20 \mathrm{wt} \%$ 乙二醇, $60 \mathrm{wt} \%$ 水) 到 PET 基底上。转印过程包括三个步骤: 玻璃基底上供墨 $\rightarrow$ 印章接触上墨 $\rightarrow$ 转移油墨, 如 图 1(b)所示。首先, 在玻璃片上滴加 $200 \mu \mathrm{L}$ 的银纳 米粒子油墨, 然后通过棒涂的方法在玻璃片上刮涂 得到均匀薄的墨层。由于印章 PDMS 的疏水特性, 当印章上墨时油墨无法在其表面铺展，因此需先经 过 $\mathrm{O}_{2}$ 等离子体处理使其亲水。然后, PDMS 印章与 玻璃片上的墨层接触 $10 \mathrm{~s}$ 后分开, 这样油墨就转移 到印章上并在其表面形成薄的墨膜。最后, PDMS 印章与加热的基底在一定压力下接触 $60 \mathrm{~s}$ 使油墨转 移到基底上，当印章与基底分离时，在 PET 基底上 就得到转移的图案。基底加热的主要目的是使印章 上的油墨与基底接触时迅速固着, 否则油墨在一定 转印压力下容易发生流动而使线条图案并糊, 无法 真实还原印章图案尺寸。

\section{3 图案的表征}

采用激光共聚焦显微镜(VK-X100K, Keyence) 和扫描电子显微镜(SU8020, Hitachi)对制备的图案 进行观测。采用方阻仪(RTS-9, 广州四探针科技有 限公司)测试网格图案的方阻, 用光纤光谱仪 (UV-1601, Shimadzu)测试其透光率。用接触角测试 系统(OCA20, Dataphysics)测试水、二碘甲烷对基底 和印章的静态接触角, 用表面张力仪 (K100, Dataphysics) 测试油墨的静态表面张力。

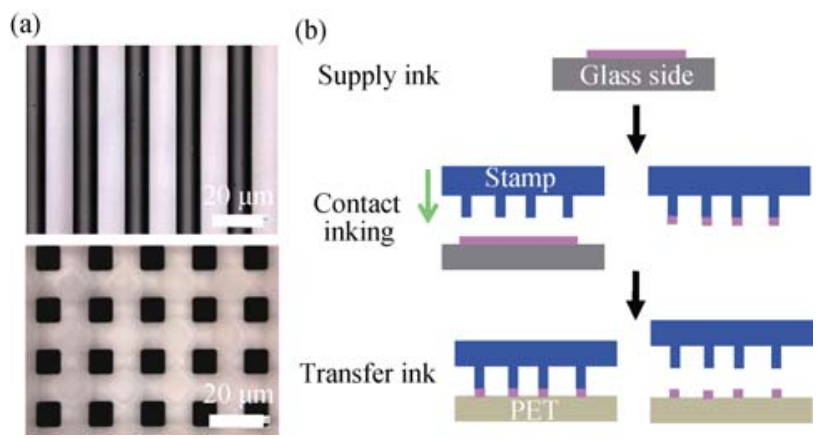

图 1 (a)用于转印的线条结构和网格结构印章图片(间距 $10 \mu \mathrm{m}$ 、 宽 $10 \mu \mathrm{m})$ 和(b)微接触印刷导电油墨过程

Fig. 1 (a) Optical image of the used PDMS stamp (10 $\mu \mathrm{m}$ width and $10 \mu \mathrm{m}$ space) and (b) $\mu \mathrm{CP}$ process of conductive ink 


\section{2 结果与讨论}

\section{1 采用线条结构印章转印图案}

\subsection{1 印章处理时间对线宽的影响}

基于前期研究 ${ }^{[19]}$, 采用线条结构的印章转印导电 油墨时, 设定基底温度为 $60^{\circ} \mathrm{C}$ 、转印压力为 $6 \sim 7 \mathrm{kPa}$ 。 转移水基银纳米粒子导电油墨时, 控制印章表面的墨 层对于实现图案的真实还原至关重要。由于 PDMS 疏水的特征, 必须先用 $\mathrm{O}_{2}$ 等离子体处理 PDMS 印 章。在 $\mathrm{O}_{2}$ 等离子体处理过程中, 高压放电产生的 高能离子、自由基等与 PDMS 之间发生多种物理 和化学反应，使印章表面引入羟基和羧基等含氧 极性基团，同时印章表面因等离子体刻蚀而变得 粗粘，二者协同效应使 PDMS 印章表面表现出较 好的亲水性, 使用的水基银纳米粒子导电油墨可 在其表面铺展。

当印章在 $200 \mathrm{~W}$ 功率下处理不同时间, 表现出 不同的表面能，如表 1 所示。表面能可通过测试水、 二碘甲烷对印章的接触角 $\theta_{1} 、 \theta_{2}$, 然后利用 Owen 公式 $\left(\gamma_{1}(1+\cos \theta)=2\left(\gamma_{1}^{\mathrm{p}} \gamma_{\mathrm{s}}^{\mathrm{p}}\right)^{1 / 2}+2\left(\gamma_{1}^{\mathrm{d}} \gamma_{\mathrm{s}}^{\mathrm{d}}\right)^{1 / 2}\right)$ 计算得到。

未经处理的印章的接触角分别为 $113.8^{\circ} 、 92.6^{\circ}$, 表面能为 $46.1 \mathrm{~mJ} / \mathrm{m}^{2}$, 而水基的银导电油墨的表面 张力是 $40.5 \mathrm{mN} / \mathrm{m}$, 两者很接近, 因此油墨无法在 印章表面润湿铺展。当 $\mathrm{O}_{2}$ 等离子体处理 $5 \mathrm{~s}$ 时, 接触 角分别降低为 $68.7^{\circ}$ 和 $54.5^{\circ}$, 表面能为 $155.9 \mathrm{~mJ} / \mathrm{m}^{2}$, 远大于油墨的表面张力, 这样导电油墨可润湿印章 并在其表面铺展。转印的线条宽为 $9.34 \mu \mathrm{m}$, 接近于 印章设计尺寸, 如图 2(a)所示。进一步延长 $\mathrm{O}_{2}$ 等离 子体处理时间, 印章的接触角不断减小而表面能增 大。当 $\mathrm{O}_{2}$ 等离子体处理时间为 $15 \mathrm{~s}$ 以上时, 表面 能稳定, 达到 $240 \mathrm{~mJ} / \mathrm{m}^{2}$ 左右, 这样接触上墨时油墨 更容易在印章表面铺展, 从而使线条变宽。从图 2(c) 可以看出, 转印的线宽为 $13.68 \mu \mathrm{m}$, 相对于印章设 计尺寸增加了 $30 \%$ 。因此, 对于本实验, 印章经过

表 $1 \mathrm{O}_{2}$ 等离子体处理不同时间的 PDMS 印章的接触角和 表面能

Table 1 Contact angle and surface energy of PDMS after $\mathrm{O}_{2}$ plasma treatment for different time

\begin{tabular}{cccc}
\hline$t / \mathrm{s}$ & $\theta_{1} /\left(^{\circ}\right)$ & $\theta_{2} /\left(^{\circ}\right)$ & $W /\left(\mathrm{mJ} \bullet \mathrm{m}^{-2}\right)$ \\
\hline 0 & 113.8 & 92.6 & 46.1 \\
5 & 68.7 & 54.5 & 155.9 \\
10 & 53.8 & 53.7 & 190.0 \\
15 & 36.5 & 53.3 & 236.4 \\
20 & 34.8 & 53.0 & 241.7 \\
\hline
\end{tabular}

(a)

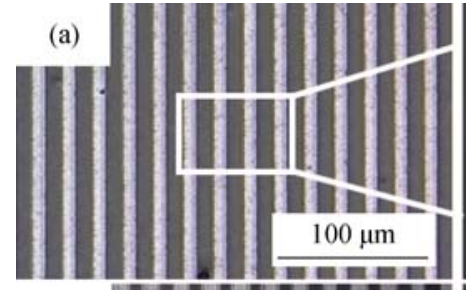

(b)
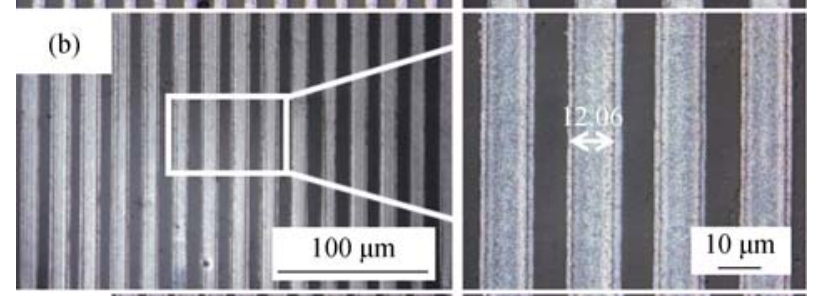

(c)
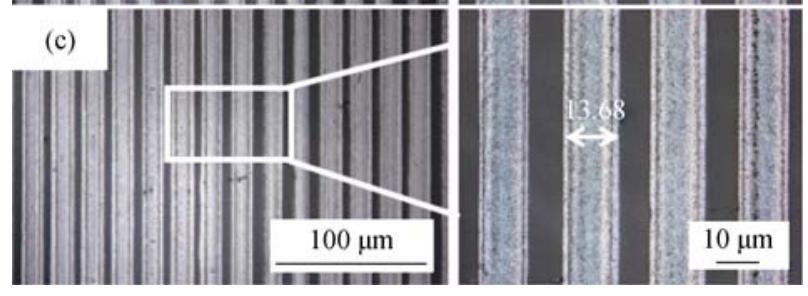

图 2 等离子体处理印章不同时间后转印的线条图案

Fig. 2 Line patterns at different treating time of stamp via $\mathrm{O}_{2}$ plasma

(a) $5 \mathrm{~s}$; (b) $10 \mathrm{~s}$; (c) $15 \mathrm{~s}$

合适的 $\mathrm{O}_{2}$ 等离子体时间 $(5 \mathrm{~s})$ 处理后，可真实还原印章 的设计尺寸。

\subsection{2 两次交叉转印制备网格图案}

通过两次交叉转印的方法, 实现线条结构印章 制备网格图案, 即在第一次转印的线条图案干燥后, 将印章旋转 $90^{\circ}$, 然后再进行印章上墨和转移油墨。 采用 $10 \mu \mathrm{m}$ 宽、不同间距 $(10 、 20 、 40 \mu \mathrm{m})$ 线条结构 的印章, 通过两次交叉转移的方法, 获得如图 3 所 示的网格图案。转印的线条边缘光滑, 即使是 $10 \mu \mathrm{m}$ 的小间距线条结构印章转印的边缘也光滑, 不会出 现并糊现象。同时, 由于第二次转印是在原先转印 的油墨干燥后再进行的, 属于湿压干的工艺, 在交 叉处两次转印的油墨之间不会互相融合而会叠加, 使交叉处墨层较厚, 这有利于提高导电性。

\section{2 采用网格结构印章转印图案}

基于上述研究, 网格结构印章也用 $\mathrm{O}_{2}$ 等离子体 处理 $5 \mathrm{~s}$, 然后转印银导电油墨。在接触上墨过程中, 由于表面张力作用油墨会在印章表面形成薄层墨膜, 包括网格之间的空隙处。干燥时，空隙处的墨膜会 发生破裂形成小孔，随后孔不断生长扩大，在网格 之间形成液桥，该液桥聚集在网格边缘，和印章凸 起部分上的油墨同时转印到基底上，如图 4 所示。

由于油墨形成的液桥的内聚力 $F$ 与液桥之间相 邻线之间的距离 $G$ 有关 ${ }^{[21]}$, 因此液桥的断裂依赖于 印章的间距。当间距较小时 $(10 \mu \mathrm{m})$, 液桥的内聚力 

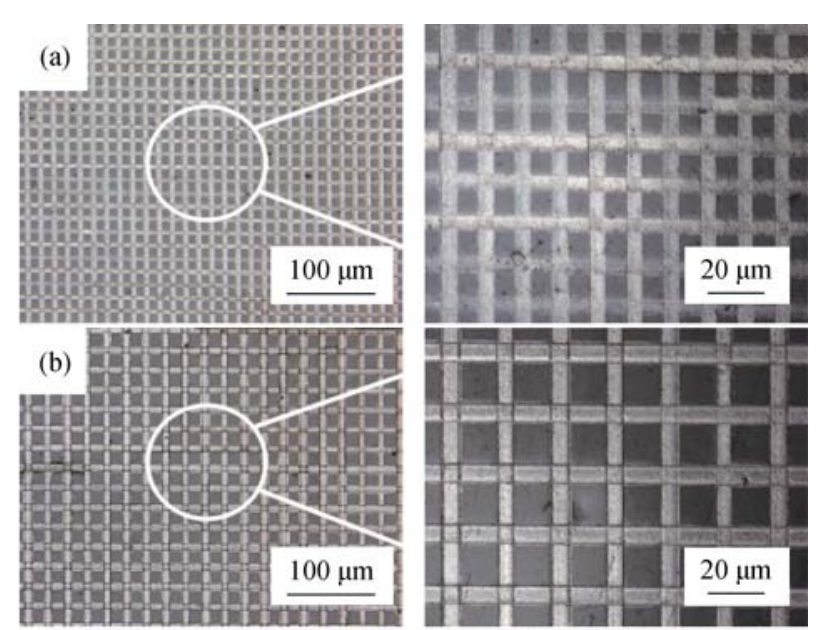

(c)
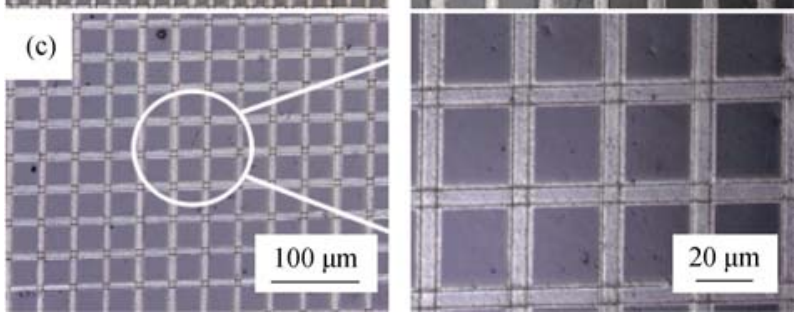

图 3 采用不同间距的印章两次交叉转印的网格图案

Fig. 3 Grid patterns using PDMS stamp with different spaces when two times transfering

(a) $10 \mu \mathrm{m}$; (b) $20 \mu \mathrm{m}$; (c) $40 \mu \mathrm{m}$

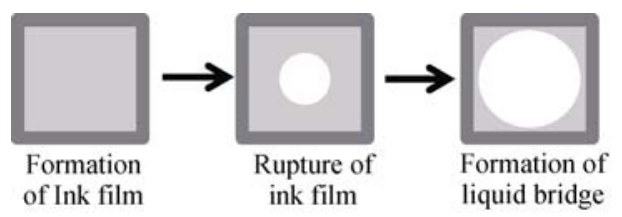

图 4 网格空隙处形成的墨膜破裂形成液桥过程

Fig. 4 Rupture process of thin ink film over the depressed hole of the stamp and formation of liquid bridge

较大, 液桥不会发生断裂, 并在表面张力作用下变 为圆弧状, 印刷的网格图案呈带弧度的孔, 而非设 计时的方形孔, 如图 5(a)所示, 并且线条宽度比原先 设计的要宽一些。当间距为 $20 \mu \mathrm{m}$ 时, 液桥仍未发生 断裂, 转印的图案类似于间距 $10 \mu \mathrm{m}$ 的结果, 边缘 也有弧度。当间距增大到 $40 \mu \mathrm{m}$ 时, 由于液桥的内聚 力下降导致其断裂, 这样来自液桥的油墨就会聚集 在印章网格的边缘, 与印章凸起部分的油墨一起转 印, 获得的线条边缘光滑无弧度, 如图 5(c)所示, 线 宽为 $13 \mu \mathrm{m}$, 比设计尺寸稍大。这是由于转印的墨层 除了印章凸起部分外, 还有印章空隙处的油墨。因此, 当使用网格结构的印章时, 印章的间距对网格图案 的形状有较大影响, 本实验合适的间距为 $40 \mu \mathrm{m}$ 。

\section{3 两种结构印章转印图案的性能对比}

采用光纤光谱仪和四探针方阻仪分别测试了两 种方法制备的网格图案的透光率和方阻。从图 6 可
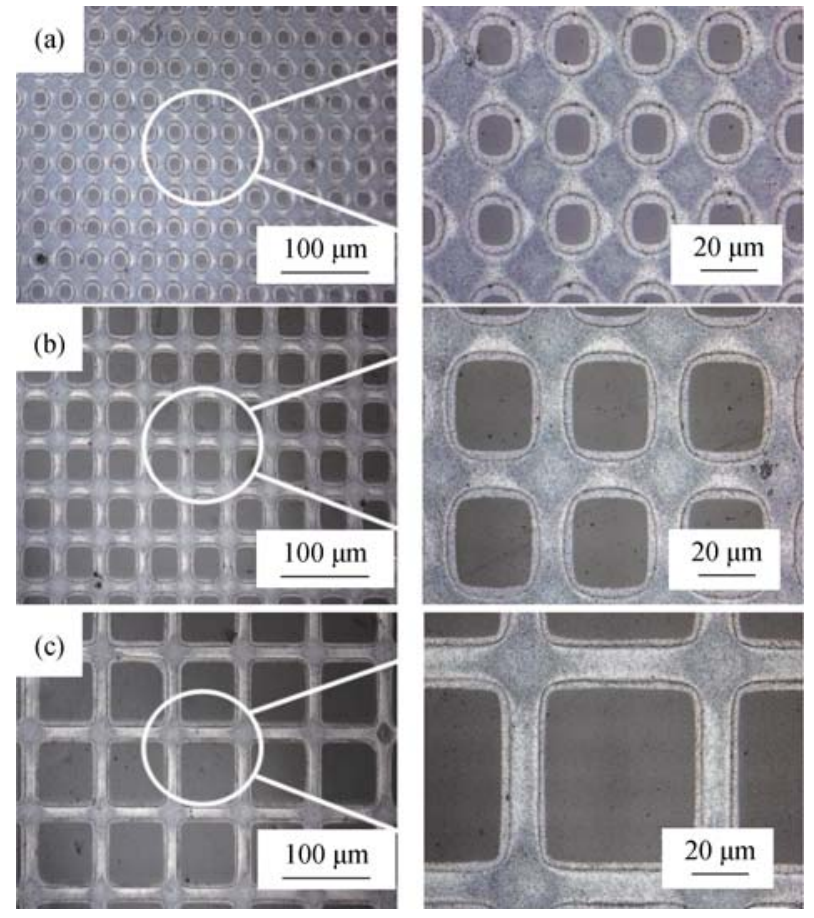

图 5 采用不同间距的网格印章转印的图案

Fig. 5 Morphologies of transferred patterns using grid-structured stamp with different size holes

(a) $10 \mu \mathrm{m}$; (b) $20 \mu \mathrm{m}$; (c) $40 \mu \mathrm{m}$

以看出, 对于相同线条间距的印章, 采用线条结构 印章进行两次交叉转印获得的图案的透光率比采用 网格结构印章获得的图案的透光率要高。当用间距 为 $10 \mu \mathrm{m}$ 网格结构印章直接印刷时, 在 $550 \mathrm{~nm}$ 处的 透光率为 $42 \%$, 而采用线条结构印章两次交叉转 印获得的图案透光率为 $54 \%$ 。这是由于采用线条结 构印章时，墨层的铺展相对较小，线条宽度和印章 设计宽度接近; 而采用网格结构印章直接印刷网 格线条时，线条边缘会发生扩印，降低了透光率。 此外, 两种方法转印时, 透光率都会随着间距的增 大而增加。

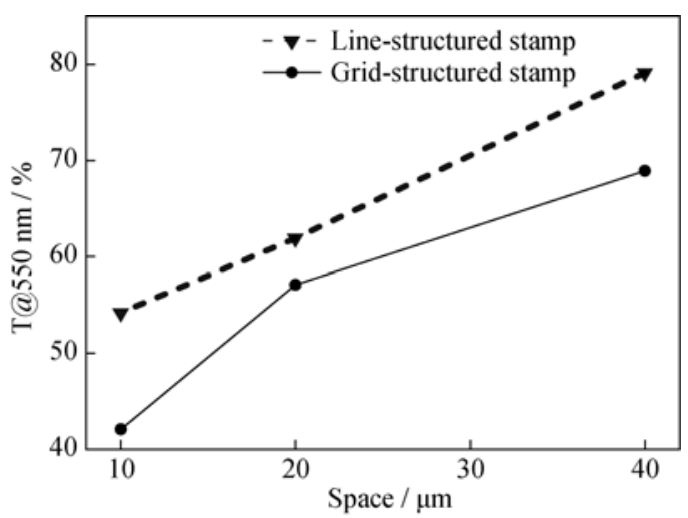

图 6 两种结构的印章转印的网格图案的透光率对比

Fig. 6 Transmittance comparison of grid patterns prepared with line- and grid-structured stamp 
从图 7 可以看出, 方阻随着间距的增大而增加, 即间距增加后导电性变差, 这主要是由于间距增大 后单位面积内的导电路径减少引起的。当采用小间 距印章时 $(10 、 20 \mu \mathrm{m})$, 两种方法得到的图案导电性 接近, 方阻分别为 $240 、 360 \Omega / \square$ 左右。这可能是由 于印章间距小时, 虽然两次交叉转印时交叉处墨层 相对较厚, 但转印得到的线条宽度比一次转印的要 窄好多, 这就削弱了交叉处墨层厚度对方阻的直接 影响。而印章间距增大为 $40 \mu \mathrm{m}$ 时, 两次交叉转印 的导电性要好, 方阻为 $420 \Omega / \square$, 比直接印的要低 $200 \Omega / \square$ 左右, 这是由于两次交叉转印得到的线宽 接近, 都是 $13 \mu \mathrm{m}$ 左右, 交叉处墨层厚度对导电性 影响较大。

因此, 采用线条结构印章可真实还原印章设计 尺寸, 避免了线条的扩展, 从而有利于透光率的提 高。同时，在网格交叉处墨层较厚有利于导电性的 提高。

\section{3 结论}

分别采用线条结构和网络结构的印章, 通过微 接触印刷方法在 PET 基底上制备了 10 13 $\mu \mathrm{m}$ 线宽的 网格状的导电图案。采用线条结构印章时, 通过优化 $\mathrm{O}_{2}$ 等离子体处理 PDMS 印章时间, 实现线条的精确 转印; 在此基础上, 通过两次交叉转印得到网格图 案, 网格线条宽度与设计尺寸接近, 并且线条间距 对图案形状几乎没有影响。采用网格结构印章转印 时，在印章表面的墨膜破裂形成的液桥易在网格边 缘处聚集并形成有弧度的图案。由于液桥的断裂依 赖于网格线条间距，只有间距较大 $(>40 \mu \mathrm{m})$ 时, 方 可去除弧度得到边缘光滑的网格图案，但线宽会增 加 30\%。采用线条结构印章可真实还原印章图案尺

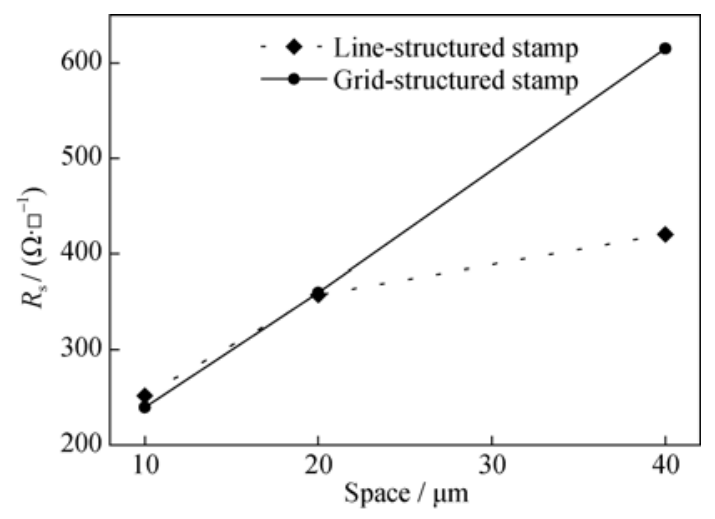

图 7 两种结构的印章转印的网格图案的导电性对比

Fig. 7 Conductivity comparison of grid patterns prepared with line- and grid-structured stamp
寸，避免了线条的扩展，有利于获得性能较好的网 格图案, 但两次转移过程相对复杂, 需用时间较长。

\section{参考文献:}

[1] ZHOU L S, WANGA A, WU S C, et al. All-organic active matrix flexible display. Appl. Phys. Lett., 2006, 88(8): 083502-1-3.

[2] VUORINEN T, ZAKRZEWSKI M, RAJALA S, et al. Printable, transparent, and flexible touch panels working in sunlight and moist environments. Adv. Funct. Mater, 2014, 24(40): 6340-6347.

[3] LIPOMI D J, TEE B C K, VOSGUERITCHIAN M. et al. Stretchable organic solar cells. Adv. Mater, 2011, 23(15): 1771-1775.

[4] TOKUNO T, NOGI M, KARAKAWA M, et al. Fabrication of silver nanowire transparent electrodes at room temperature. Nano Res., 2011, 4(12): 1215-1222.

[5] LAYANI M, GRUCHKO M, MILO O, et al. Transparent conductive coatings by printing coffee ring arrays obtained at room temperature. ACS Nano, 2009, 3(11): 3537-3542.

[6] KIM Y, NA J, PARK C, et al. PEDOT as a flexible organic electrode for a thin film acoustic energy harvester. ACS Appl. Mater. Inter., 2015, 7(30): 16279-16286.

[7] WU Q, XU Y, YAO Z, et al. Supercapacitors based on flexible graphene/polyaniline nanofiber composite films. ACS Nano, 2010, 4(4): 1963-1970.

[8] DEGANELlO D, CHERRY J A, GETHIN D T, et al. Patterning of micro-scale conductive networks using reel-to-reel flexographic printing. Thin Solid Films, 2010, 518(21): 6113-6116.

[9] PARK J, NGUYEN H A D, PARK S, et al. Roll-to-roll gravure printed silver patterns to guarantee printability and functionality for mass production. Current Applied Physics, 2015, 15(3): 367-376.

[10] OH S C, YANG K Y, BYEON K J, et al. Various metallic nano-sized patterns fabricated using an $\mathrm{Ag}$ ink printing technique. Electron Mater. Lett., 2012, 8(5): 485-489.

[11] KUMAR A, BIEBUYCK H A, WHITESIDES G M. Patterning self-assembled monolayers: applications in materials science. Langmuir, 1994, 10(5): 1498-1511.

[12] YAN X, YAO J, LU G, et al. Microcontact printing of colloidal crystals. J. Am. Chem. Soc., 2004, 126(34): 10510-10511.

[13] KIM L, ANIKEEVA P O, COE-SULLIVAN S A, et al. Contact printing of quantum dot light-emitting devices. Nano Lett., 2008, 8(12): 4513-4517.

[14] HIDBER P C, HELBIG W, KIM E, et al. Microcontact printing of palladium colloids: micron-scale patterning by electroless deposition of copper. Langmuir, 1996, 12(5): 1375-1380.

[15] TANG J, PANG W H, REN H, et al. Rapid transfer of Au nanoparticle pattern onto ITO substrate using microcontact printing technique. Acta Phys. - Chim. Sin., 2013, 29(3): 612-618. 
[16] KUSAKA Y, MIYASHITA K, USHIJIMA H. Extending microcontact printing for patterning of thick polymer layers: semi-drying of inks and contact mechanisms. J. Micromech. Microeng., 2014, 24(12): 125019-1-10.

[17] KINA O, KOUTAKE M, MATSUOKA K, et al. Organic thin-film transistors fabricated by microcontact printing. Jpn. J. Appl. Phys., 2010, 49(1):01АB07-1-4.

[18] KUSAKA Y, NOMURA K, FUKUDA N, et al. Microcontact patterning of conductive silver lines by contact inking and its layertransfer mechanisms. J. Micromech. Microeng., 2015, 25(5):
055022-1-9.

[19] LIU S, XIN Z, LI X, et al. Conductive microwires of silver nanoparticles prepared by microcontact printing. J. Nano. Res., 2016, 37(1): 36-41.

[20] ZHANG Z, ZHANG X, XIN Z, et al. Synthesis of monodisperse silver nanoparticles for ink-jet printed flexible electronics. Nanotechnology, 2011, 22(42): 425601-1-8.

[21] XIN Z, SU B, WANG J, et al. Continuous microwire patterns dominated by controllable rupture of liquid films. Small, 2013, 9(5): 722-726. 